\title{
Monotonicity and comparison results for conformal invariants
}

\author{
Albert Baernstein II and Alexander Yu. Solynin
}

\section{Dedicated to our friend Walter K. Hayman}

\begin{abstract}
Let $a_{1}, \ldots, a_{N}$ be points on the unit circle $\mathbb{T}$ with $a_{j}=e^{i \theta_{j}}$, where $0=\theta_{1} \leq \theta_{2} \leq \cdots \leq \theta_{N}=2 \pi$. Let $\Omega=\overline{\mathbb{C}} \backslash\left\{a_{1}, \ldots, a_{N}\right\}$ and let $\Omega^{*}$ be $\overline{\mathbb{C}}$ with the $n$-th roots of unity removed. The maximal gap $\delta(\Omega)$ of $\Omega$ is defined by $\delta(\Omega)=\max \left\{\theta_{j+1}-\theta_{j}: 0 \leq j \leq N-1\right\}$. How should one choose $a_{1}, \ldots, a_{N}$ subject to the condition $\delta(\Omega) \leq 2 \pi / n$ so that the Poincaré metric $\lambda_{\Omega}(0)$ of $\Omega$ at the origin is as small as possible? In this paper we answer this question by showing that $\lambda_{\Omega}(0)$ is minimal when $\Omega=\Omega^{*}$. Several similar problems on the extremal values of the harmonic measures and capacities are also discussed.
\end{abstract}

\section{Introduction}

Let $\mathbb{T}$ denote the unit circle $|z|=1$ in the complex plane $\mathbb{C}$. Write $\overline{\mathbb{C}}=\mathbb{C} \cup\{\infty\}$. Take an integer $n \geq 3$ and let $a_{1}, \ldots, a_{n}$ be points of $\mathbb{T}$. Set $\Omega=\overline{\mathbb{C}} \backslash\left\{a_{1}, \ldots, a_{n}\right\}$, and let $\lambda_{\Omega}$ denote the (density of) the Poincaré metric of $\Omega$.

How should one choose $a_{1}, \ldots, a_{n}$ to make $\lambda_{\Omega}(0)$ as large as possible? Making a domain smaller makes its Poincaré metric larger, so making its complement larger makes its Poincaré metric larger. This leads one to conjecture the inequality

$$
\lambda_{\Omega}(0) \leq \lambda_{\Omega^{*}}(0)
$$

where

$$
\Omega^{*}=\overline{\mathbb{C}} \backslash\left\{e^{\frac{2 \pi i j}{n}}: 1 \leq j \leq n\right\} .
$$

In [19] it was proved that the inequality above indeed does hold. Thus, the problem of maximizing $\lambda_{\Omega}(0)$ over all allowable $a_{1}, \ldots, a_{n}$ has been solved.

There are various related extremal problems. For example, if we take $\Omega$ to be $\mathbb{C} \backslash\left\{a_{1}, \ldots, a_{n}\right\}$ instead of $\overline{\mathbb{C}} \backslash\left\{a_{1}, \ldots, a_{n}\right\}$, will the maximal $\lambda_{\Omega}(0)$ again be achieved

Mathematics Subject Classification (2010): Primary 30C; Secondary 31A.

Keywords: Comparison theorem, hyperbolic metric, harmonic measure, capacity. 
when the $a_{1}, \ldots, a_{n}$ are equally spaced on $\mathbb{T}$ ? To the best of our knowledge, this problem remains unsolved.

Here is another problem. Let $a_{1}, \ldots, a_{N}$ be points of $\mathbb{T}$ with $a_{j}=e^{i \theta_{j}}$, where $0=\theta_{0}<\theta_{1} \leq \cdots \leq \theta_{N-1} \leq \theta_{N}=2 \pi$. Then the $\theta_{j}$ generate a partition of $[0,2 \pi]$. Define the ordered $N+1$-tuple $\Theta$ to be

$$
\Theta=\left(\theta_{0}, \theta_{1}, \ldots, \theta_{N-1}, \theta_{N}\right)
$$

and define the maximal gap $\delta(\Omega)$ of the partition by

$$
\delta(\Omega)=\max \left\{\theta_{j+1}-\theta_{j}: 0 \leq j \leq N-1\right\} .
$$

Assume that

$$
\delta(\Omega) \leq \frac{2 \pi}{n} .
$$

Then $N \geq n$, and when the $a_{j}$ are $n$ equally spaced points on $\mathbb{T}$, the gap constraint is satisfied with equality. Define $\Omega=\overline{\mathbb{C}} \backslash\left\{a_{1}, \ldots, a_{N}\right\}$, and let again $\Omega^{*}$ be $\overline{\mathbb{C}}$ with the $n$-th roots of unity removed.

Problem. How should one choose $a_{1}, \ldots, a_{N}$ so that $\lambda_{\Omega}(0)$ is as small as possible?

In this article we will solve this problem by showing that $\lambda_{\Omega}(0)$ is minimal when $\Omega=\Omega^{*}$, that is, when $N=n$ and the $a_{j}$ are equally spaced. This Poincaré metric comparison theorem will be obtained as a consequence of more general results involving PDEs of the form

$$
\Delta u=\gamma(u)+f \quad \text { in } \Omega,
$$

with boundary values

$$
u=\operatorname{constant} A \text { on } \partial \Omega,
$$

where $\gamma$ is an increasing function on $\mathbb{R}, f$ is an $n$-fold symmetric function satisfying a suitable monotonicity condition, and the open set $\Omega$ is $n$-fold symmetric. Here, $n$-fold symmetry of $\Omega$ means that $z \in \Omega$ if and only if $e^{\frac{2 \pi i}{n}} z \in \Omega$ and $z \in \Omega$ if and only if $\bar{z} \in \Omega$. A function $f$ is said to be $n$-fold symmetric if and only if $f\left(z e^{\frac{2 \pi i}{n}}\right)=f(z)$ and $f(z)=f(\bar{z})$ for all relevant $z$.

In Theorem 1 , we consider the case $n=1$ and prove that $u$ satisfies a monotonicity condition on circles. Theorem 2 is like Theorem 1, but now all the relevant objects are $n$-fold symmetric.

Theorem 3 is the main result of the paper. We start with an $n$-fold symmetric function $u$ satisfying the PDE, a partition $\Theta$ of $[0,2 \pi]$ and an $n$-fold symmetric $\Omega$. We then define a new open set, called $\Omega_{\Theta}$, and a new function $u_{\Theta}$ defined on $\Omega_{\Theta}$. These new objects are called transplants of the originals via $\Theta$. They are defined in Section 3, after the proof of Theorem 2. It is proved in Theorem 3 that if the maximal gap of $\Omega$ is less than or equal to $\frac{2 \pi}{n}$ and if $v$ is a function satisfying $\Delta v \leq \gamma(v)+f_{\Theta}$ in $\Omega_{\Theta}$ and $v \geq A$ on $\partial \Omega_{\Theta}$, then $v \geq u_{\Theta}$ in $\Omega_{\Theta}$.

When $\Omega$ is not a bounded subset of $\mathbb{C}$, we have to add some assumptions involving the behavior of $u$ at $\infty$. 
In Theorems 4-7, we use Theorem 3 to prove transplantation inequalities involving harmonic measure, hyperbolic capacity, logarithmic capacity and Poincaré metrics. The theorems have corollaries which give explicit estimates for these conformal invariants. The result discussed above about minimizing $\lambda_{\Omega}(0)$ when $\Omega=\overline{\mathbb{C}} \backslash\left\{a_{1}, \ldots, a_{N}\right\}$ is a corollary to Theorem 7 .

The results in this paper about Poincaré metrics are similar in spirit to the results in [4].

\section{A monotonicity theorem}

Let $\Omega$ be be an open set in $\mathbb{C}$ which is circularly symmetric with respect to $\mathbb{R}_{+}=$ $\{t \in \mathbb{R}: 0 \leq t<\infty\}$. This means that 0 might or might not belong to $\Omega$, and that for each $r>0$ the intersection of $\Omega$ with the circle $|z|=r$ is either empty, the whole circle, or a single arc of the form $\left\{r e^{i \theta}: 0 \leq|\theta|<\varphi(r)\right\}$, where $0<\varphi(r) \leq \pi$. For short, we shall say simply that $\Omega$ is circularly symmetric. Also, set

$$
K=\mathbb{C} \backslash \Omega, \quad \Omega^{+}=\Omega \cap\{z \in \mathbb{C}: \operatorname{Im} z>0\} .
$$

A function $f$ on $\Omega$ is said to be symmetric decreasing if $f(z)=f(\bar{z})$ for all $z \in \Omega$, and $f\left(r e^{i \theta}\right)$ is a decreasing (=nonincreasing) function of $\theta$ on the intersection of the circle $|z|=r$ with $\Omega^{+}$. Symmetric increasing functions are defined analogously.

Let now $u: \mathbb{C} \rightarrow \mathbb{R}$ be a function satisfying the following assumptions:

(a) $u \in C^{2}(\Omega \backslash\{0\}), \quad u \in C(\mathbb{C})$.

(b) $\Delta u(z)=p(|z|)[\gamma(u(z))+f(z)], \quad z \in \Omega \backslash\{0\}$, where $\gamma$ is a nonnegative continuous increasing function on $\mathbb{R}, f$ is a nonnegative continuous symmetric decreasing function on $\Omega$, and $p$ is a nonnegative continuous function on $[0, \infty)$.

(c) $u(z)=A, z \in K$, where $A$ is a finite real constant.

If $\Omega$ is unbounded, we assume also that

(d) $\lim \sup _{z \rightarrow \infty, z \in \Omega} u(z) \leq A$.

(e) There is a symmetric increasing function $u_{0}\left(r e^{i \theta)}\right)$, defined for sufficiently large $r$ and all $\theta$, such that

$$
\lim _{r \rightarrow \infty}\left[u\left(r e^{i \theta}\right)-u_{0}\left(r e^{i \theta}\right)\right]=0
$$

uniformly for $\theta \in[-\pi, \pi]$.

Theorem 1. Suppose that $\Omega$ is a circularly symmetric open set in $\mathbb{C}$, and that the function $u$ satisfies conditions (a)-(e). Then $u$ is symmetric increasing on $\mathbb{C}$.

Theorem 1 is a variant of a theorem of A. Weitsman [22]. See also Lemma 9.8 of [11]. L. E. Fraenkel's book [7] contains results like Theorem 1 involving Steiner symmetrization. 
Proof of Theorem 1. Since $\gamma, f$ and $p$ are nonnegative, $u$ is subharmonic in $\Omega \backslash\{0\}$. Since $u$ is continuous on $\mathbb{C}$ with $u=A$ on $K \backslash\{0\}$ and $u$ satisfies assumption (d) at $\infty$, it follows from the extended maximum principle [12], that $u \leq A$ everywhere in $\mathbb{C}$. Moreover, in each connected component of $\Omega$, either $u(z)<A$ for all $z$ or $u \equiv A$ in that component. In the latter case $u$ is constant in an annulus, disk, or exterior disk containing the component and thus is symmetric increasing in that annulus or disk. From now on, we shall assume that $u(z)<A$ everywhere in $\Omega$.

Let $\mathbb{H}$ denote the upper half plane $\operatorname{Im} z>0$ and write $\overline{\mathbb{H}}=\mathbb{H} \cup \mathbb{R}$. Define a function $u_{*}: \overline{\mathbb{H}} \backslash\{0\} \rightarrow \mathbb{R}$ by

$$
u_{*}\left(r e^{i \theta}\right)=\inf _{E} \int_{E} u\left(r e^{i t}\right) d t
$$

where the infimum is taken over all measurable sets $E \subset[-\pi, \pi]$ with $|E|=2 \theta$. Here, $|E|$ denotes the one-dimensional Lebesgue measure of $E$. Then for each $z=$ $r e^{i \theta}$ there exists a set $E=E(z)$ with measure $2 \theta$ such that the infimum is attained. We will call such an $E$ a minimal set. For general functions $h \in L^{1}[-\pi, \pi]$, minimal sets with $|E|=2 \theta$ have the form $E=E_{1} \cup E_{2}$, where $E_{1}=\{t \in[-\pi, \pi]: h(t)<s\}$ and $E_{2}$ is a subset of $\{t \in[-\pi, \pi]: h(t)=s\}$, chosen so that $\left|E_{1}\right|+\left|E_{2}\right|=2 \theta$.

For facts about $u_{*}$, see [11]. A key fact about $u_{*}$ for us is the inequality

$$
\int_{-\pi}^{\pi} u_{*}\left(r e^{i \theta}+\rho e^{i \psi}\right) d \psi \leq \int_{E} d t \int_{-\pi}^{\pi} u\left(r e^{i t}+\rho e^{i \psi}\right) d \psi .
$$

Here, we assume that $z=r e^{i \theta} \in \mathbb{H}$ is given, and that $\rho$ is positive and sufficiently small. This is inequality (9.5.10) of [11], where one may find a proof.

From (2.1) and $\int_{E} u\left(r e^{i t}\right) d t=u_{*}\left(r e^{i \theta}\right)$, it follows that

$$
\int_{-\pi}^{\pi}\left[u_{*}\left(r e^{i \theta}+\rho e^{i \psi}\right)-u_{*}\left(r e^{i \theta}\right)\right] d \psi \leq \int_{E} d t \int_{-\pi}^{\pi}\left[u\left(r e^{i t}+\rho e^{i \psi}\right)-u\left(r e^{i t}\right)\right] d \psi
$$

Inequality (2.2) is valid for all $r e^{i \theta} \in \mathbb{H}$ and all positive sufficiently small $\rho$.

Next, for real functions $h$ in a plane domain $D$, define the "generalized Laplacian" $\Delta h$ by

$$
\Delta h(z)=\liminf _{\rho \rightarrow 0} 4 \rho^{-2}\left\{\frac{1}{2 \pi} \int_{-\pi}^{\pi} h\left(z+\rho e^{i \psi}\right) d \psi-h(z)\right\}, \quad z \in D .
$$

If $h$ is $C^{2}$ in a disk $B\left(z, \rho_{0}\right)$ and $0<\rho<\rho_{0}$, then an application of Green's Theorem gives (see [21], or page 694 of [11])

$$
\frac{1}{2 \pi} \int_{-\pi}^{\pi} h\left(z+\rho e^{i \psi}\right) d \psi-h(z)=\frac{1}{2 \pi} \int_{|\zeta|<\rho} \Delta h(z+\zeta) \log |\rho / \zeta||d \zeta|^{2} .
$$

From the continuity of $\Delta h$ at $z$, it follows that the liminf in (2.3) exists as a limit, and that the generalized Laplacian of $h$ at $z$ equals the classical Laplacian of $h$ at $z$. 
Returning now to our function $u$, write $\Omega^{+}=\Omega \cap \mathbb{H}$, and take $z=r e^{i \theta} \in \Omega^{+}$. Then $0<\theta<\varphi(r)$. We are assuming that $u<A$ in $\Omega$, so that $u\left(r e^{i t}\right)<A$ on $|t|<\varphi(r)$ and $u\left(r e^{i t}\right)=A$ for $\varphi(r) \leq|t| \leq \pi$. Also, $u$ is continuous. A simple argument left to the reader shows existence of a minimal set $E=E(z)$ which is contained in $[-\varphi(r)+\epsilon, \varphi(r)-\epsilon]$ for some $\epsilon>0$. Thus, there exists $\rho_{0}>0$ such that the set of $\zeta$ in $\mathbb{C}$ at distance $\leq \rho_{0}$ from the set $r E$ is contained in $\Omega$. Since $u \in C^{2}(\Omega),(2.2)$ and (2.4) imply that for $\rho<\rho_{0}$,

$$
\int_{-\pi}^{\pi}\left[u_{*}\left(r e^{i \theta}+\rho e^{i \psi}\right)-u_{*}\left(r e^{i \theta}\right)\right] d \psi \leq\left.\int_{E} d t \int_{|\zeta|<\rho} \Delta u\left(r e^{i t}+\zeta\right) \log |\rho / \zeta| d \zeta\right|^{2} .
$$

Divide both sides by $\rho^{2}$. The integral with respect to $|d \zeta|^{2}$ is a bounded function of $t \in E$ and $\rho<\rho_{0}$. Thus, when $\rho \rightarrow 0$ we can move the limit on the right inside the $d t$ integral. This gives

$$
\Delta u_{*}\left(r e^{i \theta}\right) \leq \int_{E} \Delta u\left(r e^{i t}\right) d t, \quad z \in \Omega^{+} .
$$

Next, define $U: \overline{\mathbb{H}} \backslash\{0\} \rightarrow \mathbb{R}$ by

$$
U\left(r e^{i \theta}\right)=\int_{-\theta}^{\theta} u\left(r e^{i t}\right) d t
$$

Then $U$ is continuous on $\overline{\mathbb{H}} \backslash\{0\}, U \in C^{2}\left(\Omega^{+}\right)$, and a short calculation shows that

$$
\Delta U(z)=\int_{-\theta}^{\theta} \Delta u\left(r e^{i t}\right) d t, \quad z \in \Omega^{+} .
$$

Define

$$
w(z)=U(z)-u_{*}(z), \quad z \in \overline{\mathbb{H}} \backslash\{0\} .
$$

Then $w$ is continuous on $\overline{\mathbb{H}} \backslash\{0\}$. Write $E=E(z)=E\left(r e^{i \theta}\right)$ and $I=[-\theta, \theta]$. Then from (2.5), (2.6) and the PDE satisfied by $u$, we obtain

$$
\begin{aligned}
\Delta w(z) \geq & \int_{I} \Delta u\left(r e^{i t}\right) d t-\int_{E} \Delta u\left(r e^{i t}\right) d t \\
= & p(r) \int_{I} \gamma\left(u\left(r e^{i t}\right)\right) d t-p(r) \int_{E} \gamma\left(u\left(r e^{i t}\right)\right) d t \\
& \quad+p(r) \int_{I} f\left(r e^{i t}\right) d t-p(r) \int_{E} f\left(r e^{i t}\right) d t .
\end{aligned}
$$

Since $f$ is symmetric decreasing, we have $\int_{I} f\left(r e^{i t}\right) d t-\int_{E} f\left(r e^{i t}\right) d t \geq 0$. Since $\gamma$ is increasing and $E$ is a minimal set for $u$, it follows that $E$ is also a minimal set for $\gamma(u)$, so that $\int_{I} \gamma\left(u\left(r e^{i t}\right)\right) d t-\int_{E} \gamma\left(u\left(r e^{i t}\right)\right) d t \geq 0$. Hence $w$ is subharmonic in $\Omega^{+}$.

Strictly speaking, our argument shows that $w$ is a generalized subharmonic function on $\Omega^{+}$. However, a theorem of Radó (see page 14 of [16]) shows that generalized subharmonic functions coincide with ordinary subharmonic functions (defined in terms of mean values.) 
Now take $z=r e^{i \theta} \in \overline{\mathbb{H}}$ with $z \neq 0$ and $z \notin \Omega^{+}$. Recall that $\varphi(r)=\varphi$ is twice the length of the intersection of $\Omega$ with the circle $|z|=r$. Thus $\varphi(r) \leq \theta \leq \pi$. Since $u\left(r e^{i t}\right) \leq A$ for $|t| \leq \varphi(r)$ and $u\left(r e^{i t}\right)=A$ for $|t| \geq \varphi(r)$, it follows that the interval $I=[-\theta, \theta]$ is a minimal set. This implies $u_{*}\left(r e^{i \theta}\right)=U\left(r e^{i \theta}\right)$, so that $w(z)=0$ for all $z \in K \backslash\{0\}$ with $z \in \overline{\mathbb{H}}$.

From the continuity of $u$ at $z=0$, one easily checks that

$$
\lim _{z \rightarrow 0} w(z)=0
$$

If $\Omega$ is unbounded, then assumption (e) and the inequality $\int_{-\theta}^{\theta} u_{0}\left(r e^{i t}\right) \leq$ $\int_{E} u_{0}\left(r e^{i t}\right) d t$ when $|E|=2 \theta$ imply that

$$
\limsup _{z \rightarrow \infty, z \in \mathbb{H}} w(z) \leq 0 \text {. }
$$

We have shown that the continuous function $w$ is subharmonic in $\Omega^{+}$, has upper limit zero as $z \in \Omega^{+}$approaches $\partial \Omega^{+}$, and has nonpositive upper limit as $z$ approaches $\infty$. By the maximum principle, $w \leq 0$ in $\overline{\mathbb{H}}$. But the definitions of $u_{*}$ and $U$ show that $w \geq 0$. We conclude that $u_{*}=U$ in $\mathbb{H}$, which, by a simple argument, implies that $u$ is symmetric increasing on $\mathbb{C}$.

\section{A monotonicity theorem and a comparison theorem}

It is convenient to revise our notation. In this section $n$ will denote a positive integer. $K$ will denote a closed subset of $\mathbb{C}$ which is circularly symmetric with respect to $\mathbb{R}_{+}$and is contained in the closed sector

$$
|\arg z| \leq \frac{\pi}{n}
$$

Write $E=K \cap(0, \infty)$. Then $K$ has the form

$$
\left\{z=r e^{i \theta} \in \mathbb{C}: r \in E,|\theta| \leq \varphi(r)\right\},
$$

where $\varphi$ is a lower semicontinuous function on $E$ with values in $\left[0, \frac{\pi}{n}\right]$. We shall refer to such $K$ as circularly symmetric closed subsets of $|\arg z| \leq \frac{\pi}{n}$.

Define

$$
K_{n}=\bigcup_{j=0}^{n-1} e^{\frac{2 \pi i j}{n}} K
$$

where $e^{i \theta} K$ denotes rotation of $K$ through an angle $\theta$ about the origin.

For a given $K$, write

$$
\Omega_{n}=\mathbb{C} \backslash K_{n}
$$

Then $K_{n}$ is an $n$-fold symmetric closed set and $\Omega_{n}$ is an $n$-fold symmetric open set. If $K$ intersects a circle $|z|=r$ then $K_{n}$ contains all the points $z=r e^{\frac{2 \pi i j}{n}}$. If $\varphi(r)=\frac{\pi}{n}$, then $K_{n}$ is the whole circle $|z|=r$, and $\Omega_{n}$ will be disconnected unless its intersection with at least one of the sets $|z|<r$ or $|z|>r$ is empty. 
Let now $u: \mathbb{C} \rightarrow \mathbb{R}$ be a function satisfying the following assumptions:

(aa) $u \in C^{2}\left(\Omega_{n} \backslash\{0\}\right), \quad u \in C(\mathbb{C})$.

(bb) $\Delta u(z)=\gamma(u(z))+f(z), z \in \Omega_{n} \backslash\{0\}$, where $\gamma$ is a nonnegative continuous increasing function on $\mathbb{R}$ and $f$ is a nonnegative continuous $n$-fold symmetric function on $\Omega_{n}$ which is increasing on $0 \leq \theta \leq \frac{\pi}{n}$ for each fixed $r$.

(cc) $u(z)=A, z \in K_{n}$, where $A$ is a finite real constant.

If $\Omega_{n}$ is unbounded, we assume also that

(dd) $\lim \sup _{z \rightarrow \infty, z \in \Omega_{n}} u(z) \leq A$.

(ee) There is an $n$-fold symmetric function $u_{0}\left(r e^{i \theta}\right)$, defined for sufficiently large $r$ and all $\theta$, such that $u_{0}$ is decreasing on $0 \leq \arg z \leq \frac{\pi}{n}$ and

$$
\lim _{r \rightarrow \infty}\left[u\left(r e^{i \theta}\right)-u_{0}\left(r e^{i \theta}\right)\right]=0,
$$

uniformly for $\theta \in[-\pi, \pi]$.

Theorem 2. Suppose that $\Omega_{n}$ and $K_{n}$ are as above, and that the function $u$ satisfies conditions (aa)-(ee). Then $u$ is $n$-fold symmetric on $\mathbb{C}$ and $u$ is decreasing in the sector $0 \leq \theta \leq \frac{\pi}{n}$ for each fixed $r$.

Proof. Define $w(z)=u\left(e^{\frac{2 \pi i}{n}} z\right)-u(z)$. Then $w$ is continuous on $\mathbb{C}, w=0$ on $K_{n}$, and, by condition (ee), $w(z) \rightarrow 0$ as $z \rightarrow \infty$. Suppose that $w$ is somewhere positive. Then there exists $z_{0} \in \Omega_{n}$ such that $w\left(z_{0}\right)=\max _{\mathbb{C}} w>0$. There is a neighborhood $D$ of $z_{0}$ in which $w>0$. Using the symmetry of $f$, we have

$$
\Delta w(z)=\Delta u\left(e^{\frac{2 \pi i}{n}} z\right)-\Delta u(z)=\gamma\left(u\left(e^{\frac{2 \pi i}{n}} z\right)\right)-\gamma(u(z)), \quad z \in D .
$$

Since $\gamma$ is increasing, it follows that $w$ is subharmonic in $D$. Since $w$ achieves its maximum in $D, w$ must be constant in $D$. Let $D_{1}$ be the connected component of $\Omega_{n}$ which contains $z_{0}$ and $D_{2}$ be the subset of $D_{1}$ on which $w=w\left(z_{0}\right)$. The argument just given shows that $D_{2}$ is an open set. Continuity of $w$ implies that $D_{2}$ is also a relatively closed subset of $D_{1}$. Thus, $D_{2}=D_{1}$, so that $w \equiv w\left(z_{0}\right)$ in $D_{1}$. But $w=0$ on $\partial D_{1}$, so we have a contradiction. We conclude that $w \leq 0$ in $\mathbb{C}$. By the same argument, $w \geq 0$ in $\mathbb{C}$, so that $u\left(z e^{\frac{2 \pi i}{n}}\right)=u(z)$ everywhere in $\mathbb{C}$. A similar argument shows that $u(\bar{z})=u(z)$ everywhere in $\mathbb{C}$. We have shown that $u$ is $n$-fold symmetric in $\mathbb{C}$.

To prove the monotonicity statement, define the function $\tilde{u}$ on $\mathbb{C}$ and the open set $\tilde{\Omega}$ by

$$
\tilde{u}(z)=u\left(e^{\frac{i \pi}{n}} z^{1 / n}\right), \quad \tilde{\Omega}=\left\{z \in \mathbb{C}: e^{\frac{i \pi}{n}} z^{1 / n} \in \Omega_{n}\right\} .
$$

Then $\tilde{\Omega}$ is circularly symmetric, like the open sets in Section 2 . The symmetry properties of $u$ imply that $\tilde{u}$ is well defined, continuous and has the same smoothness properties as $u$. Moreover, setting $p(t)=\frac{1}{n^{2}} t^{\frac{2}{n}-2}$, it follows that $\tilde{u}$, in $\tilde{\Omega}$, satisfies the PDE

$$
\Delta \tilde{u}(z)=p(|z|) \Delta u\left(e^{\frac{\pi i}{n}} z^{1 / n}\right)=\gamma(\tilde{u}(z))+\tilde{f}(z),
$$


where $\tilde{f}(z)=f\left(e^{\frac{i \pi}{n}} z^{1 / n}\right)$. Thus, $\tilde{u}$ satisfies hypothesis (b) of Theorem 1 . It is easy to verify that the other hypotheses are also satisfied. Thus, $\tilde{u}$ is symmetric increasing on $\mathbb{C}$. This implies that $u$ is decreasing on $0 \leq \arg z \leq \frac{\pi}{n}$.

Next, for a positive integer $N$ we denote by $\Theta$ a partition of the interval $[0,2 \pi]$ into $N$ subintervals. More formally, $\Theta$ denotes an ordered $N+1$ tuple: $\Theta=$ $\left(\theta_{0}, \ldots, \theta_{N}\right)$, where $0=\theta_{0}<\theta_{1} \leq \cdots \leq \theta_{N}=2 \pi$. Define also

$$
\delta(\Theta)=\max _{1 \leq k \leq N} \theta_{k}-\theta_{k-1}
$$

We shall call $\delta(\Theta)$ the maximal gap of the partition $\Theta$. When $N=n$ and each $\theta_{k}=\frac{2 \pi k}{n}$, we will denote the corresponding partition by $\Theta^{*}$ and call it the uniform ( $n$-fold) partition.

For a circularly symmetric closed subset $K$ of $|\arg z| \leq \frac{\pi}{n}$ and partition $\Theta$ of $[0,2 \pi]$ define sets $K_{\Theta}$ and $\Omega_{\Theta}$ by

$$
K_{\Theta}=\bigcup_{k=1}^{N} e^{i \theta_{k}} K, \quad \Omega_{\Theta}=\mathbb{C} \backslash K_{\Theta} .
$$

In particular,

$$
K_{\Theta}=K_{n}
$$

is the union of $n$ equally spaced copies of $K$.

Let $g$ be an $n$-fold symmetric continuous function defined on $\mathbb{C}$. Recall that this means that $g(z)=g(\bar{z})$ and $g\left(z e^{\frac{2 \pi i}{n}}\right)=g(z)$ for every $z \in \mathbb{C}$. Let $\Theta$ be a partition with

$$
\delta(\Theta) \leq \frac{2 \pi}{n}
$$

Then $N \geq n$. We will call this inequality the maximal gap inequality.

Define the function $g_{\Theta}$ on $\mathbb{C}$ as follows: Take $z=r e^{i \theta} \in \mathbb{C}$ with $z \neq 0$ and $0 \leq \theta \leq 2 \pi$. Then $\theta_{k-1}<\theta \leq \theta_{k}$ for some $k \in\{1, \ldots, N\}$. For fixed $k$, let $m$ be the midpoint of the interval $\left[\theta_{k-1}, \theta_{k}\right]$. If $\theta_{k-1} \leq \theta \leq m$, define

$$
g_{\Theta}\left(r e^{i \theta}\right)=g\left(r e^{i\left(\theta-\theta_{k-1}\right)}\right),
$$

and if $m \leq \theta \leq \theta_{k}$, define

$$
g_{\Theta}\left(r e^{i \theta}\right)=g\left(r e^{i\left(\theta-\theta_{k}\right)}\right)
$$

Define $g_{\Theta}(0)=g(0)$.

The symmetries of $g$ imply that $g_{\Theta}$ is well defined and continuous on $\mathbb{C}$. If $g$ is symmetric decreasing on $|\theta| \leq \frac{\pi}{n}$ then $g_{\Theta}$ decreases on $\left[\theta_{k-1}, m\right]$ and increases on $\left[m, \theta_{k}\right]$. Moreover, $g_{\Theta}\left(r e^{i \theta_{k-1}}\right)=g_{\Theta}\left(r e^{i \theta_{k}}\right)=g(r)$. Thus, $g$ and $g_{\Theta}$ have the same maxima on circles $|z|=r$, but $g$ will in general have smaller minima than $g_{\Theta}$.

If $g$ is symmetric increasing the statements above change in a straightforward way. For example, $g$ and $g_{\Theta}$ have the same minima on circles $|z|=r$, but $g$ has larger maxima.

We shall call $K_{\Theta}, \Omega_{\Theta}$ and $g_{\Theta}$ the transplants of $K, \Omega$ and $g$ with respect to $\Theta$. 
With $K$ and $\Theta$ as above, suppose that $v \in C^{2}\left(\Omega_{\Theta}\right)$ is a function satisfying

$$
\Delta v \leq \gamma(v)+f_{\Theta}, \quad \text { in } \quad \Omega_{\Theta},
$$

and

$$
\liminf _{z \rightarrow \partial \Omega_{\Theta}} v(z) \geq A
$$

where $\gamma, f$ and $A$ are as in (bb) and (cc).

Here now is our comparison theorem.

Theorem 3. Let $u$ and $v$ satisfy assumptions (aa)-(ee) and (3.2), (3.3), respectively. If $\Omega$ is unbounded, assume also that at least one of the following inequalities holds:

$$
\begin{aligned}
& \limsup _{z \rightarrow \infty, z \in \Omega_{\Theta}}\left(u_{\Omega_{\Theta}}(z)-v(z)\right) \leq 0, \quad \text { or } \\
& \limsup _{z \rightarrow \infty, z \in \Omega_{\Theta}}\left(u_{\Omega_{\Theta}}(z)-v(z)\right)<\sup _{z \in \Omega_{\Theta}}\left(u_{\Omega_{\Theta}}(z)-v(z)\right) .
\end{aligned}
$$

Then

$$
u_{\Omega_{\Theta}}(z) \leq v(z), \quad \text { for all } z \in \Omega_{\Theta} .
$$

If also $\gamma$ is locally Lipschitz on $\mathbb{R}$ and equality occurs in (3.5) at some point $z_{0} \in \Omega_{\Theta}$, then, up to a rotation of $\Omega_{\Theta}, v$ coincides with $u$ in the connected component of $\Omega_{\Theta}$ which contains $z_{0}$.

In particular, if $0 \in \Omega$ then $v(0) \geq u(0)$, and the equality statement is in force.

Another way to write the conclusion (3.5) for $r e^{i \theta} \in \Omega_{\Theta}$ is

$$
u\left(r e^{i\left(\theta-\theta_{k-1}\right)}\right) \leq v\left(r e^{i \theta}\right), \quad u\left(r e^{i\left(\theta-\theta_{k}\right.}\right) \leq v\left(r e^{i \theta}\right)
$$

for every $r \geq 0, k=1, \ldots, N$, where the first inequality holds for $\theta \in\left[\theta_{k-1}, m\right]$ and the second for $\theta \in\left[m, \theta_{k}\right]$. Recall that $m=\frac{1}{2}\left(\theta_{k-1}+\theta_{k}\right)$.

Proof of Theorem 3. For brevity, write

$$
U(z)=u_{\Theta}(z) .
$$

Take $z_{0}=r_{0} e^{i \psi_{0}} \in \Omega_{\Theta} \backslash\{0\}$, and take $k$ with $\theta_{k-1} \leq \psi_{0} \leq \theta_{k}$. Suppose that $\psi_{0} \in\left[\theta_{k-1}, m\right)$. Set

$$
\alpha=e^{-i \theta_{k-1}}
$$

From the definition of $U$, it follows that

$$
U(z)=u(\alpha z), \text { hence } \Delta U(z)=\Delta u(\alpha z)
$$

for all $z$ in some neighborhood of $z_{0}$. Similarly, if $\psi_{0} \in\left(m, \theta_{k}\right]$, then

$$
U(z)=u(\beta z), \text { hence } \Delta U(z)=\Delta u(\beta z)
$$

for all $z$ in some neighborhood of $z_{0}$, where $\beta=e^{-i \theta_{k}}$. 
Suppose now that $\psi_{0}=m$. Then $U$ is Lipschitz in neighborhoods of $z_{0}$ but need not be $C^{1}$. Thus, $\Delta U$ need not exist in the classical sense. As in Section 2, we work with the generalized Laplacian of $U$, which is defined, when $z=r e^{i \theta}$, by

$$
\Delta U(z)=\liminf _{\rho \rightarrow 0^{+}} \frac{4}{\rho^{2}}\left(\frac{1}{2 \pi} \int_{0}^{2 \pi} U\left(z+\rho e^{i(\theta+\varphi)}\right) d \varphi-U(z)\right) .
$$

For $z_{0}=r_{0} e^{i m}$, it follows from the monotonicity and symmetry properties of $u$ in Theorem 2 that

$$
U\left(z_{0}+\zeta\right) \geq u\left(\alpha\left(z_{0}+\zeta\right)\right)
$$

for all $\zeta$ in some neighborhhood of 0 , with equality for $\zeta=0$. Taking $\zeta=\rho e^{i(\theta+\varphi)}$, we deduce that $\Delta U\left(z_{0}\right) \geq \Delta u\left(\alpha z_{0}\right)$. Summing up, we have shown that for every $z_{0} \in \Omega_{\Theta} \backslash\{0\}$, we have

$$
\Delta U\left(z_{0}\right) \geq \Delta u\left(\alpha z_{0}\right) \quad \text { or } \quad \Delta U\left(z_{0}\right) \geq \Delta u\left(\beta z_{0}\right)
$$

according as $\psi_{0} \in\left[\theta_{k-1}, m\right]$ or $\psi_{0} \in\left[m, \theta_{k}\right]$, respectively.

Furthermore, if $0 \in \Omega$ it is easy to show that the mean value of $U$ over small circles $|z|=r$ is greater than or equal to the mean value of $u$. Thus,

$$
\Delta U(0) \geq \Delta u(0)
$$

Combined with (3.6) and assumption (b) on $u$, we obtain for all $z \in \Omega_{\Theta}$,

$$
\Delta U(z) \geq \gamma(U(z))+f_{\Theta}(z) .
$$

Next, let $w=U-v$. We want to prove that $w \leq 0$ in $\Omega_{\Theta}$. This is essentially a special case of Theorem 10.1 in [8]. For the reader's convenience, we shall supply a proof.

From (3.7) and (3.2), it follows that for all $z \in \Omega_{\Theta}$,

$$
\Delta w(z)=\Delta U(z)-\Delta v(z) \geq \gamma(U(z))-\gamma(v(z))
$$

Suppose that the open set $\Omega^{+}:=\left\{z \in \Omega_{\Theta}: w(z)>0\right\}$ is not empty. Then since $\gamma$ is an increasing function, it follows from (3.8) that $\Delta w \geq 0$ on $\Omega^{+}$, so that $w$ is a generalized subharmonic function on $\Omega^{+}$. By [16] (see page 14), $w$ is an ordinary subharmonic function on $\Omega^{+}$.

If $\zeta \in \partial \Omega^{+}$, then either $\zeta \in \Omega_{\Theta}$, so that $w(\zeta)=0$, or $\zeta \in \partial \Omega_{\Theta}$, so that $u(\zeta)=A$ and $v(\zeta) \geq A$. So if $\Omega$ is bounded then $\lim \sup w(z) \leq 0$ when $z \rightarrow \partial \Omega^{+}$. By the maximum principle, $w \leq 0$ in $\Omega^{+}$. This contradicts the definition of $\Omega^{+}$, so we must indeed have $w \leq 0$ in $\Omega_{\Theta}$. If $\Omega$ is unbounded, then with the aid of (3.4), we see again that $w \leq 0$ in $\Omega_{\Theta}$. This proves the inequality (3.5) in Theorem 3 .

Proof of strict inequality. We continue to let $w=U-v$, where now we know that $w \leq 0$ in $\Omega_{\Theta}$. We need to show that in each connected component $\hat{\Omega}$ of $\Omega_{\Theta}$, either $w(\bar{z})<0$ for every $z \in \hat{\Omega}$ or else $w \equiv 0$ in $\hat{\Omega}$.

Since $U$ and $v$ are continuous on $\Omega_{\Theta}$, to prove our assertion, it will suffice to show that for each disk $D$ with $\bar{D} \subset \Omega_{\Theta}$, either $w<0$ everywhere in $D$, or $w=0$ everywhere in $D$. 
Define $h: D \rightarrow \mathbb{R}$ by

$$
h(z)=-\frac{\gamma(U(z))-\gamma(v(z))}{U(z)-v(z)}, \quad \text { when } \quad U(z) \neq v(z),
$$

and

$$
h(z)=0, \quad \text { when } \quad u(z)=V(z) .
$$

Since $U$ and $v$ are bounded on $D$ and $\gamma$ is Lipschitz on $[-a, a]$ for each $a \in \mathbb{R}$, it follows that $h$ is bounded on $D$. Also, $\gamma$ is increasing, so $h \leq 0$ in $D$. Finally, by (3.8), $w$ satisfies in $D$ the differential inequality

$$
\Delta w(z)+h(z) w(z) \geq 0 .
$$

For $w \in C^{2}(D)$, Theorem 6 in page 64 of [15] implies that for solutions $w$ of (3.9), either $w(z)<0$ for all $z \in D$ or $w(z)=0$ for all $z \in D$. Perusal of the proof of Theorem 6 shows that the same conclusion holds when $w$ is continuous in $D$ and the $\Delta$ in (3.9) is the generalized Laplacian. The proof of our Theorem 3 is complete.

\section{Application to harmonic measure}

By $\omega(z, \gamma, D)$ we shall denote the harmonic measure of a boundary set $\gamma \subset \partial D$ with respect to an open set $D$ evaluated at $z \in D$.

Let

$$
\mathbb{D}_{R}=\{z:|z|<R\}, \quad \mathbb{T}_{R}=\{z:|z|=R\},
$$

and let

$$
S_{n}(R)=\{z:|z|<R,|\arg z|<\pi / n\}, \quad \sigma_{n}(R)=\{z:|z|=R,|\arg z|<\pi / n\},
$$

with $S_{n}=S_{n}(1)$ and $\sigma_{n}=\sigma_{n}(1), n \geq 2$. As in Section 3, for a compact set $K$ in the closure of $S_{n}$ and a partition $\Theta$ of $[0,2 \pi]$, let

$$
D_{\Theta}=\mathbb{D} \backslash K_{\Theta} \text {. }
$$

Recall that $\left(D_{\Theta}\right)_{z_{0}}$ denotes the connected component of $D_{\Theta}$ which contains the point $z_{0}$.

Theorem 4. Let $K$ be a circularly symmetric compact subset of $S_{n} \cup \sigma_{n}$ with $n \geq 2$ and let $\Theta$ be a partition of $[0,2 \pi]$. If the maximal gap $\delta(\Theta)$ is $\leq 2 \pi / n$, then for $r e^{i t} \in D_{\Theta}$,

$$
\begin{array}{ll}
\omega\left(r e^{i t}, \mathbb{T}, D_{\Theta}\right) \leq \omega\left(r e^{i\left(t-\theta_{k-1}\right)}, \mathbb{T}, D_{\Theta^{*}}\right), & \text { if } \quad \theta_{k-1} \leq t \leq\left(\theta_{k}+\theta_{k-1}\right) / 2, \\
\omega\left(r e^{i t}, \mathbb{T}, D_{\Theta}\right) \leq \omega\left(r e^{i\left(\theta_{k}-t\right)}, \mathbb{T}, D_{\Theta^{*}}\right), & \text { if } \quad\left(\theta_{k}+\theta_{k-1}\right) / 2 \leq t \leq \theta_{k} .
\end{array}
$$

In particular, if $0 \in D_{\Theta^{*}}$, then

$$
\omega\left(0, \mathbb{T}, D_{\Theta}\right) \leq \omega\left(0, \mathbb{T}, D_{\Theta^{*}}\right) .
$$

If $z_{0} \in D_{\Theta}$, if $\left(D_{\Theta}\right)_{z_{0}}$ is regular for the Dirichlet problem, and if $0<$ $\omega\left(z_{0}, \mathbb{T}, D_{\Theta}\right)<1$, then equality occurs in (4.1) at $z_{0} \in D_{\Theta}$ or in (4.2) at $z_{0}=0$ if and only if $\left(D_{\Theta}\right)_{z_{0}}$ coincides with the corresponding connected component of $D_{\Theta^{*}}$. 
Proof. Assume first that $D_{\Theta^{*}}$ is regular for the Dirichlet problem and that $K$ is a compact subset of $S_{n}$. Then $D_{\Theta}$ is also regular. Indeed, $D_{\Theta}$ is constructed in such a way that for each point $z_{0} \in \partial D_{\Theta}$ and every sufficiently small $\varepsilon>0$ there are a point $z_{1} \in \partial D_{\Theta^{*}}$ and real number $\alpha$ such that the set $e^{i \alpha}\left(\partial D_{\Theta} \cap\left\{z:\left|z-z_{0}\right|<\varepsilon\right\}\right)$ is contained in the set $\partial D_{\Theta^{*}} \cap\left\{z:\left|z-z_{1}\right|<\varepsilon\right\}$. In other words, the set $\partial D_{\Theta}$ is "thicker" at its point $z_{0}$ than the set $\partial D_{\Theta}$ at its point $z_{1}$. Since $z_{1}$ is regular for the Dirichlet problem the latter implies that $z_{0}$ is also regular.

Take $u(z)=1-\omega\left(z, \mathbb{T}, D_{\Theta^{*}}\right)$ and $v(z)=1-\omega\left(z, \mathbb{T}, D_{\Theta}\right)$. Extend $u$ to $\mathbb{C}$ by setting $u=1$ on $K_{\Theta^{*}}$ and $u=0$ on the complement of the unit disk. Extend $v$ analogously. Then $u$ and $v$ are continuous on $\mathbb{C}$. The other hypotheses of Theorem 3 are also satisfied. Taking $A=1$ and $\gamma=f=0$, we obtain the inequalities stated in Theorem 4.

To prove the inequalities without the restrictions, one may use an approximation argument as follows. Assume first that $K$ is a compact subset of $S_{n}$ but $D_{\Theta *}$ is not necessarily regular. Approximate $K$ with a sequence of compact sets $K_{j}=\{z: \operatorname{dist}(z, K) \leq 1 / j\}, j=1,2, \ldots$ Then $K_{j}$ is circularly symmetric and $K_{j} \rightarrow K$ as $j \rightarrow \infty$. Let $D_{\Theta^{*}}^{j}$ and $D_{\Theta}^{j}$ be open sets constructed as above for the compact set $K_{j}$ with $j$ sufficiently large. It is well known that $D_{\Theta^{*}}^{j}$ and $D_{\Theta}^{j}$ are regular for the Dirichlet problem and

$$
\omega\left(z, \mathbb{T}, D_{\Theta^{*}}^{j}\right) \rightarrow \omega\left(z, \mathbb{T}, D_{\Theta^{*}}\right) \quad \text { and } \quad \omega\left(z, \mathbb{T}, D_{\Theta}^{j}\right) \rightarrow \omega\left(z, \mathbb{T}, D_{\Theta}\right) \quad \text { as } j \rightarrow \infty .
$$

Since $D_{\Theta^{*}}^{j}$ is regular for the Dirichlet problem and $K_{j} \subset S_{n}$ for all $j$ sufficiently large we conclude that inequalities (4.1) and (4.2) hold with $D$ replaced by $D^{j}$. The latter together with (4.3) implies that (4.1) and (4.2) hold for the set $D$ as well.

If $K \subset S_{n} \cup \sigma_{n}$, then we approximate $K$ with a sequence of compact sets $K_{j}=\left\{z \in K:|z| \leq 1-\frac{1}{j}\right\}, j=2,3, \ldots$ Then, arguing as above, we obtain the inequalities (4.1) and (4.2).

Finally, the uniqueness statement in Theorem 4 follows from the uniqueness statement in Theorem 3.

Theorem 4 expresses an intuitively obvious observation: if we keep the circumference of a round stove at constant temperature and place cooling screens of the same shape along some smaller concentric circle in such a way that the maximal gap between the centers of the screens is $\leq 2 \pi / n$, then the temperature at the center of the stove will be maximal when the gaps between the screens are as large as possible.

Now we give an explicit upper bound for the harmonic measure in terms of the logarithmic measure $\int_{E} \frac{d r}{r}$ of the circular projection $E$ of $K$ onto $\mathbb{R}_{+}$.

Corollary 1. Under the assumptions of Theorem 4 , let $0 \in D_{\Theta}$ and let $\int_{E} \frac{d r}{r}=$ $-\log \rho$ for some $0<\rho<1$, where $E=K \cap \mathbb{R}_{+}$. Then

$$
\omega\left(0, \mathbb{T}, D_{\Theta}\right) \leq \frac{2}{\pi} \arctan \frac{2 \rho^{n / 2}}{1-\rho^{n}}
$$

with equality if and only if $K=[\rho, 1]$ and $K \Theta=K_{\Theta^{*}}$. 
Inequality (4.4) easily follows from (4.2) and a generalization of Beurling's "shove theorem" for $n$ evenly spaced radii given by M. Essèn and K. Haliste [6]. It complements V. N. Dubinin's solution to Gonchar's problem (see Theorem 2.14 of [5]), which says that for $K=[\rho, 1]$, for any partition $\Theta$ of $[0,2 \pi]$ into $n$ subsets and for any integer $n \geq 2$, we have

$$
\omega\left(0, \mathbb{T}, D_{\Theta}\right) \geq \frac{2}{\pi} \arctan \frac{2 \rho^{n / 2}}{1-\rho^{n}} .
$$

A generalization of Gonchar's problem was studied by Baernstein [3], who showed that for every $0<r<1$ and every increasing convex function $\Phi:[0,1] \rightarrow \mathbb{R}$ the inequality

$$
\int_{0}^{2 \pi} \Phi\left(\omega\left(r e^{i \theta}, \mathbb{T}, D\left(\Theta_{n}\right)\right) d \theta \geq \int_{0}^{2 \pi} \Phi\left(\omega\left(r e^{i \theta}, \mathbb{T}, D\left(\Theta_{n}^{*}\right)\right) d \theta\right.\right.
$$

holds for $n=2,3$ and for $D\left(\Theta_{n}\right)$ and $D\left(\Theta_{n}^{*}\right)$ associated with any compact set $K \subset(0,1]$. In particular,the inequality

$$
\omega\left(0, \mathbb{T}, D\left(\Theta_{n}\right)\right) \geq \omega\left(0, \mathbb{T}, D\left(\Theta_{n}^{*}\right)\right)
$$

holds for every compact set $K \subset(0,1]$ and $n=2,3$.

It was conjectured in [3] that (4.5) and (4.6) remain valid for all $n \geq 4$. As far as we know, no progress has been made on these problems except for [20], where (4.6) was proved for all positive integers $n$ when $K=\left[r_{1}, r_{2}\right]$ and $0<r_{1}<r_{2} \leq 1$.

\section{Application to hyperbolic capacity}

For $R>0$, let $E$ be a compact subset of the disk $\mathbb{D}_{R}$. The open set

$$
D=\mathbb{D}_{R} \backslash E,
$$

the compact set $E$, and the unbounded closed set $\mathbb{C} \backslash \mathbb{D}_{R}$ form a condenser, in the sense of Chapter 4 of [10]. The capacity $\operatorname{cap}(E, D)$ of the condenser can be defined to be

$$
\operatorname{cap}(E, D)=\int_{D}|\nabla \omega(z)|^{2} d x d y .
$$

where $\omega(z)$ is the harmonic measure

$$
\omega(z)=\omega\left(z, \mathbb{T}_{R}, D\right) .
$$

The open set $D$ need not be connected. There is, however, a unique connected component $D^{\prime}$ of $D$ which contains $\mathbb{T}_{R}$ in its boundary.

An argument with Green's formula gives

$$
\operatorname{cap}(E, D)=\int_{0}^{2 \pi} \frac{\partial \omega}{\partial r}\left(R e^{i \theta}\right) R d \theta .
$$

When $R=1$, we call cap $(E, D)$ the hyperbolic capacity of $E$, and denote it by caph $E$. 
Let us resume now our notations $K_{\Theta}$, etc. In the theorem below, $D_{\Theta^{*}}^{\prime}$ is the connected component of $D_{\Theta^{*}}$ whose boundary contains the unit circle $\mathbb{T}$. Recall that for partitions $\Theta=\left\{\theta_{0}, \ldots, \theta_{N}\right\}$ we always take $\theta_{0}=0$ and $\theta_{N}=2 \pi$.

Theorem 5. Let $K$ be a circularly symmetric compact set in $\bar{S}_{n} \backslash \mathbb{T}$ with $n \geq 2$ and let $\Theta$ be a partition of $[0,2 \pi]$. If the maximal gap $\delta(\Theta)$ is $\leq 2 \pi / n$, then

$$
\operatorname{caph} K_{\Theta} \geq \operatorname{caph} K_{\Theta^{*}} .
$$

If $D_{\Theta^{*}}^{\prime}$ is regular for the Dirichlet problem, then equality occurs in (5.1) if and only if $D_{\Theta}^{\prime}=D_{\Theta^{*}}^{\prime}$.

Proof. Let

$$
\omega^{*}(z)=\omega\left(z, \mathbb{T}, D_{\Theta^{*}}\right), \quad \omega(z)=\omega\left(z, \mathbb{T}, D_{\Theta}\right)
$$

Then

$$
\operatorname{caph} K_{\Theta}=\int_{0}^{2 \pi} \frac{\partial \omega}{\partial r}\left(e^{i \theta}\right) d \theta, \quad \text { and } \quad \operatorname{caph} K_{\Theta^{*}}=\int_{0}^{2 \pi} \frac{\partial \omega^{*}}{\partial r}\left(e^{i \theta}\right) d \theta .
$$

In the discussion to follow, we assume that $r \in(0,1)$ is sufficiently close to 1 so that $\mathbb{T}_{r} \subset D_{\Theta^{*}}^{\prime}$. Also, we write $m_{k}=\frac{1}{2}\left(\theta_{k-1}+\theta_{k}\right)$. Then Theorem 4 implies that

$$
\omega\left(r e^{i t}\right) \leq \omega^{*}\left(r e^{i\left(t-\theta_{k-1}\right)}\right)
$$

when $t$ is such that $\theta_{k-1} \leq t \leq m_{k}$, while if $m_{k} \leq t \leq \theta_{k}$ then the $t-\theta_{k-1}$ is changed to $\theta_{k}-t$. Since $\omega=\omega^{*} \equiv 1$ on $\mathbb{T}$, it follows that

$$
\frac{\partial \omega}{\partial r}\left(e^{i t}\right) \geq \frac{\partial \omega^{*}}{\partial r}\left(e^{i\left(t-\theta_{k-1}\right)}\right)
$$

when $t$ is such that $\theta_{k-1} \leq t \leq m_{k}$, with the same change as before when $m_{k} \leq$ $t \leq \theta_{k}$. From this inequality, we deduce that

$$
\int_{0}^{2 \pi} \frac{\partial \omega}{\partial r}\left(e^{i t}\right) d t \geq 2 \sum_{k=1}^{N} \int_{0}^{\frac{\theta_{k}-\theta_{k-1}}{2}} \frac{\partial \omega^{*}}{\partial r}\left(e^{i t}\right) d t .
$$

From Theorem 2, it follows that $\omega^{*}$ is $n$-fold symmetric on circles $\mathbb{T}_{r}$ and is increasing on $0 \leq \theta \leq \frac{\pi}{n}$ for fixed $r$. It follows that $\frac{\partial \omega^{*}}{\partial r}$ is $n$-fold symmetric and is decreasing on $0 \leq \theta \leq \frac{\pi}{n}$. By assumption, $\theta_{k}-\theta_{k-1} \leq \frac{2 \pi}{n}$ for each $k$. Thus, for $1 \leq k \leq N$, we see that

$$
2 \int_{0}^{\frac{\theta_{k}-\theta_{k-1}}{2}} \frac{\partial \omega^{*}}{\partial r}\left(e^{i t}\right) d t \geq \int_{\theta_{k-1}}^{\theta_{k}} \frac{\partial \omega^{*}}{\partial r}\left(e^{i t}\right) d t .
$$

Summing (5.4) from 1 to $N$ and using (5.3), we obtain

$$
\int_{0}^{2 \pi} \frac{\partial \omega}{\partial r}\left(e^{i t}\right) d t \geq \int_{0}^{2 \pi} \frac{\partial \omega^{*}}{\partial r}\left(e^{i t}\right) d t .
$$

With (5.2), this proves the inequality statement in Theorem 5 .

To obtain the equality statement, note first that if $D_{\Theta}^{\prime}=D_{\Theta^{*}}^{\prime}$ then $\omega^{*}=\omega$, so that (5.1) holds with equality. 
To prove the converse, we make the following claim: If $D_{\Theta^{*}}^{\prime}$ is not an annulus, then $\frac{\partial \omega^{*}}{\partial r}\left(e^{i t}\right)$ is is a strictly decreasing function of $t$ on the interval $\left[0, \frac{\pi}{n}\right]$.

If $D_{\Theta^{*}}^{\prime}$ is an annulus then it is not hard to see that $D_{\Omega}^{\prime}$ is the same annulus, and thus (5.1) holds with equality. Let us assume, then, that $D_{\Theta^{*}}^{\prime}$ is not an annulus, and assume also that the claim is true.

We argue by contraposition. Suppose that the sets $D_{\Theta^{*}}^{\prime}$ and $D_{\Theta}^{\prime}$ do not coincide. Then, by an argument left to the reader, we must have $N \geq n+1$ and $\theta_{k}-\theta_{k-1}<\frac{2 \pi}{n}$ for some $k \in\{0,1, \ldots, n-1\}$. Denote the smallest such $k$ also by $k$. Then $\theta_{j}=\frac{2 \pi j}{n}$ for $0 \leq j<k$, hence $m_{k}$ can not be one of the points $\frac{2 \pi j}{n}$ with $0 \leq j \leq$ $n-1$. It follows that strict inequality holds in (5.4), and hence also holds in (5.5). By (5.2), we have strict inequality in (5.1). This completes the proof of Theorem 5 , modulo the claim.

To prove the claim, first observe that $\omega^{*}\left(e^{i t}\right)$ is non increasing on $\left[0, \frac{\pi}{n}\right]$, by Theorem 2. Thus, $\frac{\partial \omega^{*}}{\partial r}\left(e^{i t}\right)$ is nondecreasing on $\left[0, \frac{\pi}{n}\right]$. If the claim is false, there are points $0<t_{1}<t_{2}<\frac{\pi}{n}$ such that $\frac{\partial \omega^{*}}{\partial r}\left(e^{i t}\right)$ is a constant $c$ on $\left[t_{1}, t_{2}\right]$. Hopf's Lemma (see page 65 in [15]) implies that $c>0$. Take an open disk $U$ with center on the unit circle whose intersection with the unit circle is contained in the shorter $\operatorname{arc} \gamma$ from $e^{i t_{1}}$ to $e^{i t_{2}}$ and whose intersection with $\mathbb{D}$ is contained in $D_{\Theta^{*}}^{\prime}$. By the Schwarz Reflection Principle, $\omega^{*}$ has a harmonic extension to $U$. Let $\tilde{\omega}$ be a conjugate harmonic function for $\omega$ in $U$. Define

$$
h(z)=\omega^{*}(z)+i \tilde{\omega}(z)-c \log z, \quad z \in U,
$$

where $\log z=\log r+i \theta$, with $z=r e^{i \theta}$ and $0<\theta<\frac{\pi}{n}$. Then $h$ is holomorphic in $U$. Let $u=R e h$. Since $\omega^{*}$ is constant on $\mathbb{T}$, a simple calculation shows that $\frac{\partial u}{\partial \theta}=0$ on $\gamma \cap U$. Similarly, from $\frac{\partial \omega}{\partial r}=c$ on $\gamma$, it follows that $\frac{\partial u}{\partial r}=0$ on $\gamma \cap U$. Thus, $\nabla u=0$ on $\gamma \cap U$, which implies that $h^{\prime}=0$ on $\gamma \cap U$, which implies that $h$ is a constant $b$ in $U$. From the definition of $h$ and fact that $\omega^{*}=1$ on $\mathbb{T}$, one deduces that $\operatorname{Re} b=1$ and that

$$
\omega^{*}(z)=1+c \log r, \quad z \in U
$$

Since $U \cap \mathbb{D}$ is a nonempty open subset of the connected open set $D_{\Theta^{*}}^{\prime}$, the equation above still holds for all $z \in D_{\Theta^{*}}^{\prime}$. It follows that $D_{\Theta^{*}}^{\prime}$ must be an annulus. The claim is proved.

\section{Application to logarithmic capacity}

The logarithmic capacity, cap $K$, of a compact set $K \subset \mathbb{C}$ is defined by

$$
-\log \operatorname{cap} K=\lim _{z \rightarrow \infty}(g(z)-\log |z|),
$$

where $g(z)$ denotes Green's function of the unbounded component of $\overline{\mathbb{C}} \backslash K$ having singularity at $z=\infty$. See [17] or [12] for background. The logarithmic capacity can be characterize in purely geometrical terms as Fekete's transfinite diameter $d(E)$. 
Precisely, we have

$$
\operatorname{cap} K=d(E):=\lim _{n \rightarrow \infty} \max _{z_{1}, \ldots, z_{n} \in K}\left(\prod_{1 \leq k<j \leq n}\left|z_{j}-z_{k}\right|\right)^{\frac{2}{n(n-1)}} .
$$

Another useful fact about the logarithmic capacity is the following principle of symmetrization which goes back to G. Pólya and G. Szegö [14]:

Theorem A. Let $K \subset \mathbb{C}$ be a compact set and let $E^{*}$ denote either the Steiner symmetrization of $E$ with respect to the real axis or the circular symmetrization of $E$ with respect to the positive real axis. Then

$$
\operatorname{cap} K^{*} \leq \operatorname{cap} K \text {. }
$$

If the unbounded component of $\overline{\mathbb{C}} \backslash K$ is regular for the Dirichlet problem, then equality occurs in (6.3) if and only if $K^{*}$ coincides with $K$ up to a translation in the vertical direction in case of the Steiner symmetrization or up to rotation about the origin in case of the circular symmetrization.

The inequality for the transfinite diameters, which is equivalent to (6.3), can be found in [14]. As concerns the uniqueness assertion of Theorem A, it is well known to experts but we could not find a precise reference. There are several ways to prove this. For instance, one may use the uniqueness assertion of the polarization comparison theorem for Green's functions; see Theorem 1 in [18].

Theorem 6. Let $K$ be a circularly symmetric compact set in the closure of $S_{n}(\infty)$ with $n \geq 2$ and let $\Theta$ be a partition of $[0,2 \pi]$. If the maximal gap $\delta(\Theta)$ is $\leq 2 \pi / n$, then

$$
\operatorname{cap} K_{\Theta} \geq \operatorname{cap} K_{\Theta^{*}} .
$$

If $D_{\Theta^{*}}^{\prime}$ is regular for the Dirichlet problem, then equality occurs in (6.4) if and only if $D_{\Theta}^{\prime}=D_{\Theta^{*}}^{\prime}$.

Proof 1. Scaling the condensers $\left(K_{\Theta}, R\right)$ and $\left(K_{\Theta^{*}}, R\right)$ and applying Theorem 5, we find that

$$
\operatorname{cap}\left(K_{\Theta}, R\right) \geq \operatorname{cap}\left(K_{\Theta^{*}}, R\right)
$$

for all sufficiently large $R>0$.

By an asymptotic formula for the logarithmic capacity (or, equivalently, for the inner radius of a domain), see Section 4.8.2 in [10], we have

$$
-\frac{1}{2 \pi} \log \operatorname{cap} K_{\Theta}=\frac{1}{\operatorname{cap}\left(K_{\Theta}, R\right)}-\frac{1}{2 \pi} \log R+o(1) \quad \text { as } R \rightarrow \infty
$$

and

$$
\left.-\frac{1}{2 \pi} \log \operatorname{cap} K_{\Theta^{*}}=\frac{1}{\operatorname{Cap}\left(K_{\Theta^{*}}, R\right.}\right)-\frac{1}{2 \pi} \log R+o(1) \quad \text { as } R \rightarrow \infty .
$$

This together with (6.5) implies (6.4). 
We will not analyze the cases of equality in (6.4) along these lines (although this is possible) because we give below a second proof of inequality (6.4), where analysis of the cases of equality is easier.

Proof 2. Let $g$ and $g^{*}$ denote Green's functions of the domains $D_{\Theta}^{\prime}$ and $D_{\Theta^{*}}^{\prime}$, respectively, each with pole at $z=\infty$. Then $g^{*}\left(R e^{i \theta}\right)$ satisfies the symmetry conditions (ee) and for large $R>0$ strictly increases on $0 \leq \theta \leq \pi / n$ unless $D_{\Theta^{*}}^{\prime}$ is the exterior of a disk centered at $z=0$; see Proposition 5 and its corollary in [2].

Assume that $D_{\Theta}$ and $D_{\Theta^{*}}^{\prime}$ are regular for the Dirichlet problem. Extend $g$ and $g^{*}$ to $\mathbb{C}$ by setting $g=0$ outside $D_{\Theta}^{\prime}$ and $g^{*}=0$ outside $D_{\Theta^{*}}^{\prime}$. Then $g$ and $g^{*}$ are continuous in $\mathbb{C}$, and $-g^{*}$ satisfies the hypotheses (aa), (bb), (cc) and (dd) which the function $u$ satisfies in Theorem 3 , with $\gamma=f=A=0$. The argument in the proof of Theorem 3 , see (3.8), shows that $-g_{\Theta}^{*}$ is subharmonic in $D_{\Theta}$. Let $w=g_{\Theta}^{*}-g$. Then, since $g$ is harmonic in $D_{\Theta}^{\prime}$, we see that $w$ is superharmonic in $D_{\Theta}^{\prime}$. Moreover, $w=0$ on $\mathbb{C} \backslash D_{\Theta}^{\prime}$, since $g$ and $g_{\Theta}$ each have this property. Furthermore, $\lim _{z \rightarrow \infty} w(z)$ exists and is finite. It follows that $w$ has a superharmonic extension to the domain $D_{\Theta}^{\prime} \cup \infty$.

By the strong minimum principle, either $w(\infty)>0$ or $w \equiv 0$ in $\overline{\mathbb{C}}$. So by $(5.1)$, we have

$$
\operatorname{cap} K_{\Theta} \geq \operatorname{cap} K_{\Theta *}
$$

with equality if and only if $w \equiv 0$ on $\overline{\mathbb{C}}$.

If $D_{\Theta}^{\prime}=D_{\Theta^{*}}^{\prime}$ the Green functions are equal, so that cap $K_{\Theta}=\operatorname{cap} K_{\Theta^{*}}$.

Conversely, if the two capacities are equal, then $w(\infty)=0$, so that $g=g_{\Theta}^{*}$, and hence $g_{\Theta}^{*}$ is harmonic in $D_{\Theta}^{\prime}$. If $D_{\Theta}^{\prime}$ is the exterior of a disk centered at the origin, it is easy to see directly that $D_{\Theta}^{\prime}=D_{\Theta^{*}}^{\prime}$. If $D_{\Theta}^{\prime}$ does not equal $D_{\Theta^{*}}^{\prime}$ then from the harmonicity of $g^{*}$ at $\infty$ one sees that for all large $r, g^{*}\left(r e^{i \theta}\right)$, is strictly increasing for $0 \leq \theta \leq \frac{\pi}{n}$. If $\theta_{k}-\theta_{k-1}<\frac{2 \pi}{n}$ for some $k$, then the definition of $g_{\Theta}^{*}$ shows that $\frac{\partial g_{\Theta}^{*}}{\partial \theta}$ has a discontinuity at $\theta=m_{k}$. This violates harmonicity, so it must be true that the partition $\Theta$ equals $\Theta^{*}$, from which it follows that $D_{\Theta}^{\prime}=D_{\Theta^{*}}^{\prime}$.

Theorem 6 leads to some explicit lower bounds for the logarithmic capacity. With $K$ as in Theorem 6 and $l \in \mathbb{R}_{+}$, define

$$
E=K \cap \mathbb{R}_{+}, \quad I_{l}=[0, l]
$$

and write meas $(E)$ for the linear measure of $E$.

Corollary 2. Under the assumptions of Theorem 6 , suppose that meas $(E)=l>0$. Then

$$
\operatorname{cap} K_{\Theta} \geq \operatorname{cap}\left(I_{l}\right)_{\Theta^{*}}=4^{-1 / n} l,
$$

with equality if and only if $K=I_{l}$ and $K_{\Theta}=K_{\Theta^{*}}$.

Proof. By Theorem 6, we have

$$
\operatorname{cap} K_{\Theta} \geq \operatorname{cap} K_{\Theta *}
$$

with equality if and only if $D_{\Theta}^{\prime}$ coincides with $D_{\Theta^{*}}^{\prime}$. 
Since the logarithmic capacity is a monotone set function, we have

$$
\operatorname{cap} K_{\Theta^{*}} \geq \operatorname{cap} E_{\Theta^{*}}
$$

If $K$ is regular for the Dirichlet problem, then equality occurs in (6.8) if and only if $K=E$.

Now inequality (6.6), together with the statement on the equality cases, follows from (6.7), (6.8) and Lemma 1 below.

Lemma 1. Let $E$ be a compact set in $\mathbb{R}_{+}$such that meas $(E)=l>0$, and set $I_{l}=[0, l]$. Then

$$
\operatorname{cap} E_{\Theta^{*}} \geq \operatorname{cap}\left(I_{l}\right)_{\Theta^{*}} .
$$

If $E$ is regular for the Dirichlet problem, then equality occurs in (6.9) if and only if $E=I_{l}$.

Proof. In the case $n=1$, the set $I_{l}$ coincides with the Steiner symmetrization of $E$ with respect to the vertical line $\{z: \operatorname{Re} z=l / 2\}$ and in the case $n=2,\left(I_{l}\right)_{\Theta *}$ coincides with the Steiner symmetrization of $E_{\Theta^{*}}$ with respect to the imaginary axis. Thus, for $n=1,2$, Lemma 1 follows from Theorem A.

Suppose now $n \geq 3$. Let

$$
E_{1}=E_{\Theta^{*}}, \quad E_{2}=\left(I_{l}\right)_{\Theta^{*}}, \quad E_{3}=\left\{r^{n} \in \mathbb{R}_{+}: r \in E\right\}, \quad E_{4}=\left\{r^{n} \in \mathbb{R}_{+}: r \in I_{l}\right\},
$$

and let $g_{j}$ be the Green function with pole at $\infty$ for $\mathbb{C} \backslash E_{j}$, for $1 \leq j \leq 4$. Then

$$
g_{1}(z)=\frac{1}{n} g_{3}\left(z^{n}\right), \quad g_{2}(z)=\frac{1}{n} g_{4}\left(z^{n}\right), \quad z \in \mathbb{C} .
$$

Using our definition of capacity, it follows that

$$
\operatorname{cap} E_{1}=\left(\operatorname{cap} E_{3}\right)^{1 / n}, \quad \operatorname{cap} E_{2}=\left(\operatorname{cap} E_{4}\right)^{1 / n} \text {. }
$$

Now

$$
\operatorname{meas}\left(E_{3}\right)=\int_{E_{3}} d s=\int_{E} n r^{n-1} d r, \quad \operatorname{meas}\left(E_{4}\right)=\int_{I_{l}} n r^{n-1} d r=l^{n} .
$$

Since $r \rightarrow r^{n-1}$ is an increasing function on $[0, \infty)<\infty$, an easy argument with integrals shows that meas $\left(E_{3}\right)>\operatorname{meas}\left(E_{4}\right)$ unless meas $\left(E \backslash I_{l}\right)=0$. Since $E$ is compact and meas $(E)=l$ the latter equality implies that $E \supset I_{l}$.

By a well-known inequality (see, e.g., page 138 in [17]), we also have cap $\left(E_{3}\right) \geq$ $\frac{1}{4}$ meas $\left(E_{3}\right)$, with equality when $E_{3}$ is replaced by $E_{4}$. Combining these inequalities, we obtain

unless $E \supset I_{l}$ and meas $\left(E \backslash I_{l}\right)=0$.

$$
\operatorname{cap} E_{3}>\operatorname{cap} E_{4}=\frac{1}{4} l^{n}
$$

In the remaining case when $E \supset I_{l}, E \neq I_{l}$, and meas $\left(E \backslash I_{l}\right)=0$ we assume that $E$ is regular for the Dirichlet problem. Then by the maximum principle for subharmonic functions, $g_{2}(z)-g_{1}(z)>0$ for all $z \in \overline{\mathbb{C}} \backslash\left(I_{l}\right)_{\Theta^{*}}$. Using our definition of the logarithmic capacity, it follows that $\operatorname{cap}\left(E_{1}\right)>\operatorname{cap}\left(E_{2}\right)$. This completes the proof. 
For any $n \geq 1$, the inequality (6.9) is in fact known for the much more general case where we replace the uniform partition $\Theta^{*}$ with any partition $\Theta$ of the interval $[0,2 \pi]$ into $n$ disjoint subintervals; see Lemma 3 in [1].

\section{Application to the Poincaré metric}

We shall consider now another conformal invariant, the Poincaré metric, with constant curvature -4 , of a domain $D \subset \overline{\mathbb{C}}$. If the complement $\overline{\mathbb{C}} \backslash D$ contains at least three points then $D$ has the unit disc $\mathbb{D}$ as its universal covering surface. If $\Pi: \mathbb{D} \rightarrow D$ is a universal covering map, the Poincaré metric $\lambda_{D}$ for $D$ is given by

$$
\lambda_{D}(\Pi(z))\left|\Pi^{\prime}(z)\right|=1 /\left(1-|z|^{2}\right), \quad \text { where } z \in \mathbb{D}, w=\Pi(z) .
$$

The Poincaré metric can also be characterized as the maximal solution of the equation

$$
\Delta \log \lambda=4 \lambda^{2}
$$

in $D$; see [22]. For further results on the Poincaré metric and its applications we refer to Chapter 9 of [11].

Theorem 7. Let $K$ be a circularly symmetric compact set in the closure of $S_{n}(\infty)$ in $\overline{\mathbb{C}}$, let $n \geq 2$ and let $\Theta$ be a partition. If $K_{\Theta^{*}}$ contains at least three points and if $\delta(\Theta) \leq 2 \pi / n$, then for $r e^{i t} \in \Omega\left(\Theta_{N}\right)$, we have

$$
\begin{array}{ll}
\lambda_{\Omega_{\Theta}}\left(r e^{i t}\right) \geq \lambda_{\Omega_{\Theta *}}\left(r e^{i\left(t-\theta_{k-1}\right)}\right), & \text { if } \quad \theta_{k-1} \leq t \leq\left(\theta_{k}+\theta_{k-1}\right) / 2, \\
\lambda_{\Omega_{\Theta}}\left(r e^{i t}\right) \geq \lambda_{\Omega_{\Theta^{*}}}\left(r e^{i\left(\theta_{k}-t\right)}\right), \quad \text { if } \quad\left(\theta_{k}+\theta_{k-1}\right) / 2 \leq t \leq \theta_{k} .
\end{array}
$$

In particular, if $0 \in \Omega_{\Theta}$ then

$$
\lambda_{\Omega_{\Theta}}(0) \geq \lambda_{\Omega_{\Theta *}}(0) .
$$

Equality occurs in (7.1) at some point $z_{0} \in \Omega_{\Theta}$ or in (7.2) at $z_{0}=0$ if and only if the connected component $\left(\Omega_{\Theta}\right)_{z_{0}}$ coincides with the corresponding connected component of $\Omega_{\Theta^{*}}$.

Proof. If $\left(\Omega_{\Theta^{*}}\right)_{z_{0}}$ is a disk, the exterior of a disk, or an annulus centered at $z=0$, then $\left(\Omega_{\Theta}\right)_{z_{0}} \subset\left(\Omega_{\Theta^{*}}\right)_{z_{0}}$ and the conclusions of the theorem are obvious. If $\left(\Omega_{\Theta^{*}}\right)_{z_{0}}$ is one of those domains then $\Omega_{\Theta}=\Omega_{\Theta^{*}}$. So, we may exclude these cases from further consideration.

For a fixed $r>0, \lambda_{\Omega_{\Theta^{*}}}\left(r e^{i \theta}\right)$ strictly decreases in $\varphi(r)<\theta<\pi / n$. To prove this, one may use, as in Theorem 2 , the non-univalent change of variables $\zeta=z^{n}$. Then, applying polarization as in the proof of Theorem 13 in [18], we obtain the desired monotonicity.

Next we approximate $\Omega_{\Theta *}$ by a sequence of $n$-fold symmetric open sets $\left(\Omega_{\Theta^{*}}\right)_{j}=$ $\left\{z: \lambda_{\Omega_{\Theta^{*}}}(z)<j\right\}, j \geq 1$. Then $K_{j}=\left\{\overline{\mathbb{C}} \backslash \Omega_{j}:|\arg z| \leq \pi / n\right\}$ is a circularly symmetric compact set in $\overline{\mathbb{C}}$. Let $\left(K_{j}\right)_{\Theta}$ and $\left(\Omega_{j}\right)_{\Theta}$ denote the transplants of $K_{j}$, as defined just before the statement of Theorem 3. Let $\lambda_{N j}$ denote the Poincaré metric of $\left(\Omega_{j}\right)_{\Theta}$. Since $\left(\Omega_{j}\right)_{\Theta} \subset\left(\Omega_{j+1}\right)_{\Theta}$ for $j \geq 1$ and $\cup_{j=1}^{\infty}\left(\Omega_{j}\right)_{\Theta}=\Omega_{\Theta}, \lambda_{N j} \rightarrow \lambda_{\Omega_{\Theta}}$ uniformly in the spherical metric on compact subsets of $\Omega_{\Theta}$, see [13] or [22]. 
Suppose that $\Omega_{\Theta^{*}}$ is a bounded subset of $\mathbb{C}$. Then for $\left(\Omega_{\Theta^{*}}\right)_{j}$ and $\left(\Omega_{j}\right)_{\Theta}$ with $j$ sufficiently large, the hypotheses of Theorem 3 are satisfied, with $u=\log \lambda_{\Omega_{\Theta^{*}}}$, $A=j, v=\log \lambda_{N j}$, and $B=\infty$. The conclusions of Theorem 3 as $j \rightarrow \infty$ then yield (7.1) and (7.2).

Suppose now that $\Omega_{\Theta^{*}}$ is unbounded. As in the proof of Theorem 3, consider the function $w=U-v$ with $U(z)=u_{\Theta}(z)$ and $u$ and $v$ defined above. Suppose that the open set $\Omega^{+}:=\left\{z \in\left(\Omega_{j}\right)_{\Theta}: w(z)>0\right\}$ is not empty. If $\Omega^{+}$is bounded, then arguing as in the proof of Theorem 3 we will get a contradiction with our assumption $\Omega^{+} \neq \emptyset$. So we must indeed have $w \leq 0$ in $\left(\Omega_{j}\right)_{\Theta}$ in this case. If $\Omega^{+}$ is unbounded and there is a finite point $\zeta$ in the closure of $\Omega^{+}$such that

$$
\sup _{z \in \Omega^{+}} w(z)=\limsup _{z \rightarrow \zeta} w(z)>0,
$$

then our argument in the proof of Theorem 3 remains valid and we again conclude that $w \leq 0$ in the case under consideration.

It remains to consider the case when $\Omega^{+}$is unbounded and

$$
\sup _{z \in \Omega^{+}} w(z)=\limsup _{z \rightarrow \infty} w(z)>0 .
$$

To discuss this case, we change variables via $z \mapsto 1 / z$. Then we consider sets $\left(\widehat{\Omega}_{j}\right)_{\Theta}=\left\{z: z^{-1} \in\left(\Omega_{j}\right)_{\Theta}\right\},\left(\widehat{\Omega}_{\Theta^{*}}\right)_{j}=\left\{z: z^{-1} \in\left(\Omega_{\Theta *}\right)_{j}\right\}$, and $\widehat{\Omega}^{+}=\left\{z: z^{-1} \in \Omega^{+}\right\}$. Let $\hat{\lambda}_{N j}$ and $\hat{\lambda}_{\left(\Omega_{\Theta^{*}}\right)_{j}}$ denote the Poincaré metrics of $\left(\widehat{\Omega}_{j}\right)_{\Theta}$ and $\left(\widehat{\Omega}_{\Theta^{*}}\right)_{j}$, respectively. Using the well known formula for the change in the Poincaré metric under conformal mapping (see Theorem 9.10 in [11]), we obtain

$$
\lambda_{N j}\left(z^{-1}\right)=|z|^{2} \hat{\lambda}_{N j}(z), \quad \lambda_{\left(\Omega_{\left.\Theta^{*}\right)_{j}}\right.}\left(z^{-1}\right)=|z|^{2} \hat{\lambda}_{\left(\Omega_{\Theta^{*}}\right)_{j}}(z), \quad z \neq 0, \infty,
$$

Let $\hat{U}$ be the function on $\left(\widehat{\Omega}_{j}\right)_{\Theta}$ defined for the partition $\Theta$ and function $\hat{u}=$

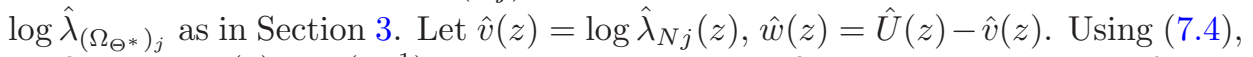
we find that $\hat{w}(z)=w\left(z^{-1}\right)$, where $w=U-v$ is defined above in this proof.

Suppose that $\infty \in \Omega^{+}$. Then $0 \in \widehat{\Omega}^{+}$. In this case, as we have shown in the proof of Theorem 3 , the function $\hat{w}$ cannot take its maximal value at $z=0$ unless $\hat{w}$ is constant on the corresponding connected component of $\widehat{\Omega}^{+}$. Since $\hat{w}(0)$ is finite and $\hat{w}(z) \rightarrow-\infty$ as $z$ approaches to the boundary of such connected component, we conclude that $\hat{w}$ cannot be constant and therefore the sets $\widehat{\Omega}^{+}$and $\Omega^{+}$must be empty in this case.

If $0 \in \partial \widehat{\Omega}^{+}$, then using the boundary conditions for $\hat{U}$ and $\hat{v}$, we find that

$$
\limsup _{z \rightarrow \infty} w(z)=\limsup _{z \rightarrow 0} \hat{w}(z)=A-B=-\infty .
$$

Since $\limsup _{z \rightarrow 0} \hat{w}(z)=\limsup _{z \rightarrow \infty} w(z)$, the latter contradicts our assumption (7.3). This contradiction implies that the set $\widehat{\Omega}^{+}$is empty and therefore the set $\Omega^{+}$is empty as well.

We have proved that $w \leq 0$ on $\Omega_{\Theta}$. As in the proof of Theorem $3, w$ satisfies for suitable $h$ a differential inequality $\Delta w+h w \geq 0$. Using again the maximum principle as in the proof of Theorem 3, we obtain the equality statement in Theorem 7 . The proof is complete. 
Theorem 7 implies sharp lower bounds for the Poincaré metric of domains obtained by removing finitely many points on the unit circle from $\mathbb{C}$ or $\overline{\mathbb{C}}$. Let $\Theta=\left(\theta_{0}, \ldots, \theta_{N}\right)$ be a partition of $[0,2 \pi]$. Set $a_{k}=e^{i \theta_{k}}$, and define

$$
\overline{\mathbb{C}}\left(a_{1}, \ldots, a_{N}\right)=\overline{\mathbb{C}} \backslash\left\{a_{1}, \ldots, a_{N}\right\}, \quad \mathbb{C}\left(a_{1}, \ldots, a_{N}\right)=\mathbb{C} \backslash\left\{a_{1}, \ldots, a_{N}\right\} .
$$

Set $e_{k}=e^{\frac{2 \pi i k}{n}}$, for $1 \leq k \leq n$.

Corollary 3. If $\delta\left(\Theta_{N}\right) \leq 2 \pi / n$, then

$$
\begin{aligned}
& \lambda_{\overline{\mathbb{C}}_{\left(a_{1}, \ldots, a_{N}\right)}}(0) \geq \lambda_{\overline{\mathbb{C}}\left(e_{1}, \ldots, e_{n}\right)}(0)=\frac{\Gamma\left(1-\frac{1}{n}\right) \Gamma\left(\frac{1}{2}+\frac{1}{n}\right)}{\Gamma\left(1+\frac{1}{n}\right) \Gamma\left(\frac{1}{2}-\frac{1}{n}\right)}, \quad n \geq 3, \\
& \lambda_{\mathbb{C}\left(a_{1}, \ldots, a_{N}\right)}(0) \geq \lambda_{\mathbb{C}\left(e_{1}, \ldots, e_{n}\right)}(0)=4^{-1 / n} \frac{\Gamma\left(1-\frac{1}{2 n}\right) \Gamma\left(\frac{1}{2}+\frac{1}{2 n}\right)}{\Gamma\left(1+\frac{1}{2 n}\right) \Gamma\left(\frac{1}{2}-\frac{1}{2 n}\right)}, \quad n \geq 2 .
\end{aligned}
$$

Equality occurs in (7.5) or (7.6) if and only if $\overline{\mathbb{C}}\left(a_{1}, \ldots, a_{N}\right)$ coincides with $\overline{\mathbb{C}}\left(e_{1}, \ldots, e_{n}\right)$ or $\mathbb{C}\left(a_{1}, \ldots, a_{N}\right)$ coincides with $\mathbb{C}\left(e_{1}, \ldots, e_{n}\right)$, respectively.

To obtain the explicit expressions on the right hand sides see, e.g., page 86 in [9]. To obtain the inequalities, apply Theorem 7 taking the compact set $K$ to be $K=\{1\}$ for $(7.5)$, and $K=\{1, \infty\}$ for (7.6).

As noted in the introduction, inequality (7.5) complements Theorem 4 in [19], which says that for every partition $\Theta$ with a fixed number $n$ of punctures, we have

$$
\lambda_{\overline{\mathbb{C}}\left(a_{1}, \ldots, a_{n}\right)}(0) \leq \lambda_{\overline{\mathbb{C}}\left(e_{1}, \ldots, e_{n}\right)}(0)=\frac{\Gamma\left(1-\frac{1}{n}\right) \Gamma\left(\frac{1}{2}+\frac{1}{n}\right)}{\Gamma\left(1+\frac{1}{n}\right) \Gamma\left(\frac{1}{2}-\frac{1}{n}\right)}, \quad n \geq 3,
$$

with equality only for the case of equally spaced points. The question about existence of a counterpart for inequality (7.6) remains open.

For our last example, take a number $\rho \in(0,1)$. Let $K$ be the union of the line segment $[\rho, 1]$ with the set $\{z \in \overline{\mathbb{C}}:|z| \geq 1,|\arg z| \leq \pi / n\}$. Then the domain $\Omega_{\Theta^{*}}$ is the unit disk minus the equally spaced line segments $e_{k}[\rho, 1]$, where $1 \leq k \leq n$. The domain $\Omega_{\Theta}$ is the open unit disk from which $N$ rays connecting the unit circle with the circle $|z|=\rho$ have been removed.

Corollary 4. With the situation above, if $\Theta$ is a partition of $[0,2 \pi]$ with $\delta(\Theta) \leq$ $2 \pi / n$, then

$$
\lambda_{\Omega_{\Theta}}(0) \geq \lambda_{\Omega_{\Theta^{*}}}(0)=\frac{\left(1+\rho^{n}\right)^{2 / n}}{4^{1 / n} \rho}
$$

with equality if and only if $K=[\rho, 1]$ and $\Omega_{\Theta}=\Omega\left(\Theta^{*}\right)$.

The domains $\Omega_{\Theta}$ are simply connected. Thus, one can calculate their Poincaré metrics in terms of derivatives of conformal maps from the unit disk. We invite the reader to use this fact to verify the equality on the right hand side above. 


\section{References}

[1] Akhmedzyanova, E. G. and Dubinin, V. N.: Radial transformations of sets, and inequalities for the transfinite diameter. Izv. Vyssh. Uchebn. Zaved. Mat. (1999), no. 4, 3-8; English transl. in Russian Math. (Iz. VUZ) 43 (1999), no. 4, 1-6.

[2] Baernstein II, A.: Integral means, univalent functions and circular symmetrization. Acta Math. 133 (1974), 139-169.

[3] Baernstein II, A.: On the harmonic measure of slit domains. Complex Variables Theory Appl. 9 (1987), 131-142.

[4] Baernstein II, A., Eremenko, A., Fryntov, A. and Solynin, A.: Sharp estimates for hyperbolic metrics and covering theorems of Landau type. Ann. Acad. Sci. Fenn. Math. 30 (2005), 113-133.

[5] Dubinin, V. N.: Symmetrization in the geometric theory of functions of a complex variable. Uspehi Mat. Nauk 49 (1994), 3-76. English transl. in Russian Math. Surveys 49 (1994), no. 1, 1-79.

[6] Essén, M. And Haliste, K.: On Beurling's theorem for harmonic measure and the rings of Saturn. Complex Variables Theory Appl. 12 (1989), no. 1-4, 137-152.

[7] Fraenkel, L. E.: Introduction to maximum principles and symmetry in elliptic problems. Cambridge Tracts in Mathematics 128, Cambridge University Press, Cambridge, 2000.

[8] Gilbarg, D. and Trudinger, N. S.: Elliptic partial differential equations of second order. Grundlehren der Mathematischen Wissenschaften 224, Springer-Verlag, Berlin-New York, 1977.

[9] Goluzin, G. M.: Geometric theory of functions of a complex variable. Translations of Mathematical Monographs 26, American Math. Society, Providence, RI, 1969.

[10] Hayman, W. K.: Multivalent functions. Second edition. Cambridge Tracts in Mathematics 110, Cambridge University Press, Cambridge, 1994.

[11] Hayman, W. K.: Subharmonic functions. Vol. 2. London Mathematical Society Monographs 20, Academic Press, London, 1989.

[12] Hayman, W. K. And Kennedy, P. B.: Subharmonic functions. Vol. 1. London Mathematical Society Monographs 9, Academic Press, London-New York, 1976.

[13] Hejhal, D.: Universal covering maps for variable regions. Math. Z. 137 (1974), 7-20.

[14] Pólya, G. And Szegö, G.: Isoperimetric inequalities in mathematical physics. Annals of Mathematics Studies 27, Princeton University Press, Princeton, NJ, 1951.

[15] Protter, M. And Weinberger, H.: Maximum principles in differential equations. Prentice-Hall, Englewood Cliffs, NJ, 1967.

[16] Radó, T.: Subharmonic functions. Chelsea, New York, 1949.

[17] Ransford, T.: Potential theory in the complex plane. London Mathematical Society Student Texts 28, Cambridge University Press, Cambridge, 1995.

[18] Solynin, A. Yu.: Functional inequalities via polarization. Algebra i Analiz 8 (1996), no. 6, 148-185. English transl. in St. Petersburg Math. J. 8 (1997), no. 6, 1015-1038.

[19] Solynin, A. Yu.: Radial projection and the Poincaré metric. Zap. Nauchn. Sem. POMI 237 (1997), 148-160. English transl. in J. Math. Sci. (New York) 95, no. 3 (1999), 2267-2275. 
[20] Solynin, A. Yu.: Harmonic measure of radial segments and symmetrization. Mat. Sb. 189 (1998) no. 11, 121-138. English transl. in Sb. Math. 189 (1998), no. $11,1701-1718$.

[21] Weitsman, A.: A symmetry property of the Poincaré metric. Bull. London Math. Soc. 11 (1979), no. 3, 295-299.

[22] Weitsman, A.: Symmetrization and the Poincaré metric. Ann. of Math. (2) 124 (1986), 159-169.

Received January 27, 2011.

Albert Baernstein II: Washington University, St. Louis, MO 63130, USA.

E-mail: al@math. wustl.edu

Alexander Y. Solynin: Texas Tech University, Lubbock, TX 79409, USA.

E-mail: alex.solynin@ttu.edu 